\title{
An Improved Equivalent Circuit Model of a Four Rod Deflecting Cavity
}

\author{
R. Apsimon ${ }^{a, b}$, G. Burt ${ }^{a, b}$ \\ ${ }^{a}$ The University of Lancaster, Lancaster LA1 4YW, United Kingdom \\ ${ }^{b}$ The Cockcroft Institute, Sci-Tech Daresbury, Daresbury, Warrington, United Kingdom
}

\begin{abstract}
In this paper we present an improved equivalent circuit model for a four rod deflecting cavity which calculates the frequencies of the first four modes of the cavity as well as the $\frac{R_{T}}{Q}$ for the deflecting mode. Equivalent circuit models of RF cavities give intuition and understanding about how the cavity operates and what changes can be made to modify the frequency, without the need for RF simulations, which can be time-consuming. We parameterise a generic four rod deflecting cavity into a geometry consisting of simple shapes. Equations are derived for the line impedance of the rods and the capacitance between the rods and these are used to calculate the resonant frequency of the deflecting dipole mode as well as the lower order mode and the model is bench-marked against two test cases; the CEBAF separator and the HL-LHC 4-rod LHC crab cavity. CST and the equivalent circuit model agree within $4 \%$ for both cavities with the LOM frequency and within $1 \%$ for the deflecting frequency. $\frac{R_{T}}{Q}$ differs between the model and CST by $37 \%$ for the CEBAF separator and $25 \%$ for the HL-LHC 4-rod crab cavity; however this is sufficient for understanding how to optimise the cavity design. The model has then been utilised to suggest a method of separating the modal frequencies in the HL-LHC crab cavity and to suggest design methodologies to optimise the cavity geometries.
\end{abstract}

Keywords: Crab cavity; equivalent circuit; LHC; 4RCC

\section{Introduction}

RF cavities operating in a dipole mode have a variety of applications in modern particle accelerators and colliders. Common uses of RF deflecting cavities are for longitudinal beam diagnostics [1], emittance exchangers [2], X-ray pulse compression [3] and crab-crossing of bunches in colliders [4, 5]. In this report we shall focus on developing an equivalent circuit model for a four rod deflecting cavity (4RDC) which operates in a TEM-110 like mode. The model is compared to the design frequencies of the CEBAF RF separator cavity [6] and the proposed HL-LHC 4-rod crab cavity [7, 8] which both show a good agreement between the equivalent circuit model and simulation results. Other deflecting cavity designs exist, such as the double quarter-wave crab cavity [9] and the RF dipole crab cavity [10]; which are also proposed for HL-LHC.

A $4 \mathrm{RDC}$ is a deflecting cavity containing four rods arranged in a plane, consisting of two parallel sections of two longitudinally opposing rods, as shown in Figure 1 . The four rods act as separate coupled quarter-wave resonators, which allows the cavity to resonate in the desired deflecting mode. However as there are four coupled resonators, there are four eigenmodes of the system due to the different permutations of the polarity of the charge on each rod. The eigenmodes are two dipole modes where transversely opposite rods have opposite charges giving a transverse field, and two monopole modes where the transversely opposing rods have the same polarity giving a longitudinal field.

Equivalent circuit models of RF cavities are a useful means of estimating cavity parameters such as the resonant frequency and $R / Q$ and give intuition and understanding about how the cavity operates and what changes can be made to modify the frequency, without the need for RF simulations which can be time-consuming. Existing equivalent circuit models for 4RDCs, such as the model outlined in [11], are based on simplifications such as neglecting the capacitance in the gap between longitudinally opposed rods and ignoring the effect of the outer can of the cavity. Ignoring the end capacitance yields the same resonant frequency for all four eigenmodes, which is incorrect. Having the lower order mode (LOM) and deflecting dipole mode at the same frequency would make it difficult to damp the unwanted monopole mode hence it is beneficial to separate the two modes in frequency. An improved equivalent circuit model would provide an understanding of how best to separate these modes.

In this report, we present an improved equivalent circuit model whereby we derive equations for the line impedance of the rods for the deflecting mode as well as the LOM. We then provide a model for the capacitance between longitudinally opposed rods, starting with a physical model and then add some empirical correction terms to create a model which fits the observed results from CST simulations [12]. We then use the line impedance and end capacitance models to determine the resonant frequencies of a 4RDC for both the LOM and deflecting mode. We then use this equivalent circuit model to develop a model for the $\frac{R_{T}}{Q}$, also known as the geometric transverse shunt impedance, for the deflecting mode of the cavity. Finally, we discuss how to use the equivalent circuit model to optimise the cavity design parameters. 


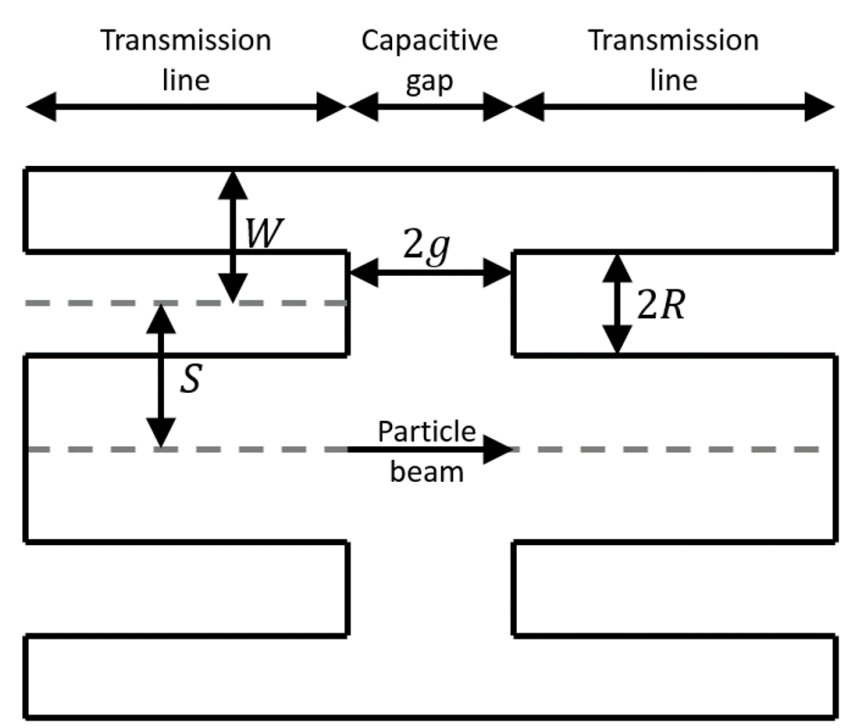

Figure 1: A cross-sectional diagram of a generic four rod deflecting cavity.

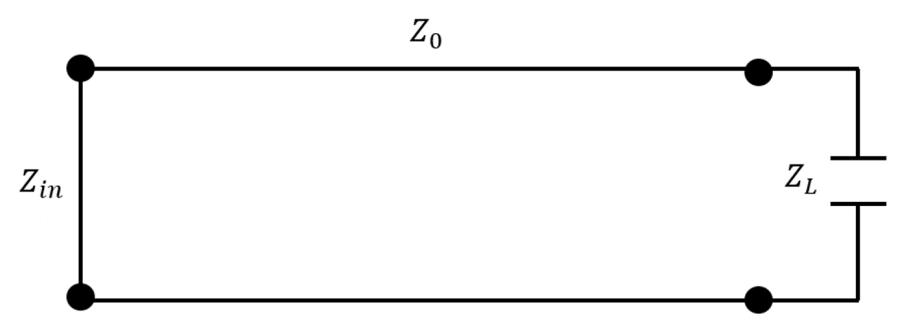

Figure 2: An equivalent circuit for a rod in a four rod deflecting cavity described as a transmission line terminated by a capacitance.

\section{Equivalent Circuit Model}

From the cross-section of a generic 4RDC (Figure 1), we may consider the cavity as consisting of three distinct regions; two transmission lines separated by a capacitive region. The geometry of the cavity has been parameterised in terms of the transverse rod separation, $2 S$, the longitudinal rod separation, $2 g$, the rod to wall separation, $W$, the rod length, $L_{\text {rod }}$ and the rod radius, $R$.

We assume that the transmission line has a characteristic impedance, $Z_{0}$, and that this is terminated through the capacitive region with a load impedance, $Z_{L}=-\frac{j}{\omega C_{\text {end }}}$, where $C_{\text {end }}$ is the capacitance between the end of the rod and the symmetry plane between longitudinally opposing rods (Figure 3), which is a ground plane. This can be expressed by the equivalent circuit shown in Figure 2 where the input impedance, $Z_{\text {in }}$, can be expressed as [13]

$$
Z_{\text {in }}=\frac{Z_{L}+j Z_{0} \tan \left(\frac{\omega L_{r o d}}{c}\right)}{Z_{0}+j Z_{L} \tan \left(\frac{\omega L_{r o d}}{c}\right)} .
$$

At the resonant frequency, the input impedance tends to zero, because the end is shorted, therefore from Eq. 1, the resonant frequency can be determined by solving [14]

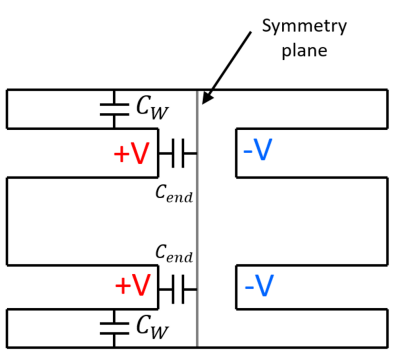

(a) Monopole (LOM)

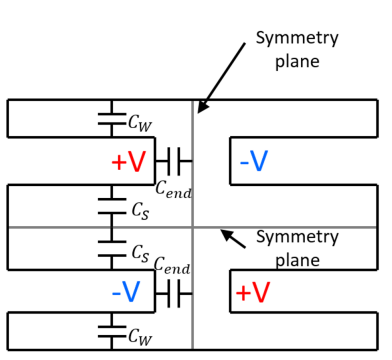

(b) Dipole
Figure 3: Diagrams illustrating the rod potential configurations for the LOM and deflecting modes respectively.

$$
\frac{1}{\omega C_{\text {end }}}=Z_{0} \tan \left(\frac{\omega L_{\text {rod }}}{c}\right) .
$$

Hence if we can determine the characteristic line impedance of the relevant mode, $Z_{0}$ and the capacitance between longitudinally opposing rods, $C_{\text {end }}$, we can calculate the resonant frequencies of the deflecting cavity. Conversely, if we neglect the capacitance between longitudinally opposed rods, as in other equivalent circuit models of $4 \mathrm{RDCs}$, from Eq. 2 we obtain $L_{\text {rod }}=\frac{\lambda}{4}$ and the frequency of all four eigenmodes becomes $\frac{c}{4 L_{\text {rod }}}$.

\subsection{Transmission line characteristic line impedance}

The configuration of rod potentials differs between the deflecting mode and the LOM for the deflecting cavity (Figure 3). The potential of the outer can and the symmetry planes can be considered as ground. In the LOM, the capacitances of the transversely opposing rods can be considered in parallel, thus the capacitance of the transmission line can be defined as $C_{\text {line }}^{L O M}=2 C_{W}$. For the deflecting mode, the capacitances of the transversely opposing rods can be considered as being in series because the rods have opposite charges; hence the capacitance of the transmission line can be defined as $C_{\text {line }}^{\text {dipole }}=$ $\frac{1}{2}\left(C_{W}+C_{S}\right)$.

In Figure 4, one can see the other monopole and dipole modes. As the longitudinally opposing rods have the same potential, there is no end capacitance in this system, however there is a small capacitance to ground between the end of the rod and the wall and transversely opposing rod (only for the dipole mode). However this capacitance is small compared to the line impedance of the rod and therefore the difference in frequency between the two eigenmodes is small. If $g$ tends to zero, the frequency of both eigenmodes tends to $\frac{c}{4 L_{r o d}}$. Furthermore these eigenmodes have very low shunt impedance compared to the other modes and can therefore be neglected.

In this paper, we consider the case where the outer can of the cavity has a rectangular cross section, such that the walls parallel to the deflecting plane are far away from the rods. This allows us to neglect image charges out of the deflecting plane as these can significantly alter the line impedance of the rods, particularly for the LOM. Image charges out of the deflecting 


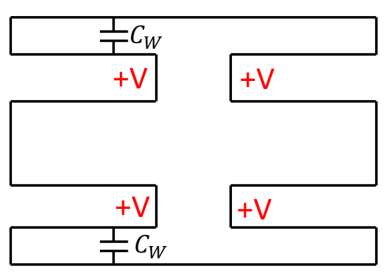

(a) Monopole

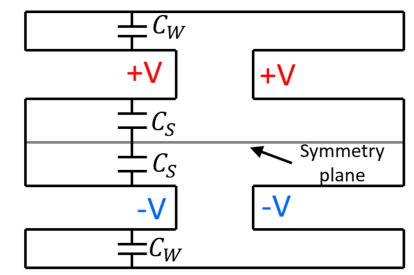

(b) Dipole
Figure 4: Diagrams illustrating the rod potential configurations for the other monopole and dipole eigenmodes.

plane can be extremely difficult to model as the distance to image charges tend to be over a continuous range rather than at discrete values, hence the perturbation to the line impedance due to these image charges cannot be expressed as a series or product expansion.

In order to determine the line impedance of the rods, we shall consider each rod as a uniform line charge, where the total charge of each rod is $q_{\text {rod }}= \pm \lambda_{\text {rod }} L_{\text {rod }}$. The potential at a transverse distance, $x$, from a uniform line charge of length $L_{\text {rod }}$ can be expressed as

$$
V=\frac{\lambda_{r o d}}{4 \pi \varepsilon_{0}} \ln \left(\frac{\sqrt{x^{2}+L_{r o d}^{2}}+L_{r o d}}{\sqrt{x^{2}+L_{r o d}^{2}}-L_{r o d}}\right)
$$

However for simplicity, we shall assume $L_{\text {rod }} \rightarrow \infty$, and the potential can be expressed as

$$
V=\frac{\lambda_{\text {rod }}}{2 \pi \varepsilon_{0}} \ln \left(\frac{\left|x_{r e f}\right|}{|x|}\right) .
$$

Where $x_{\text {ref }}$ is an arbitrary constant, which for simplicity we shall define as $x_{\text {ref }}=1$. By using the definition $|x|=\sqrt{x^{2}}$, we can show that

$$
V=\frac{\lambda_{\text {rod }}}{2 \pi \varepsilon_{0}} \ln \left(\frac{1}{|x|}\right)=\frac{\lambda_{\text {rod }}}{4 \pi \varepsilon_{0}} \ln \left(\frac{1}{x^{2}}\right) .
$$

We shall consider the potential in the upstream half of the 4RDC due to the two rods and the nearest image charge of each rod in the deflecting plane, which is assumed to be the horizontal plane. In the presence of multiple line charges, the apparent position of each line charge is displaced by a distance, $d$, such that the potential on the surface of each rod remains uniform. For the LOM, the potential due to the two rods and two image charges can be expressed as

$$
\begin{gathered}
V_{L O M}=\frac{\lambda_{\text {rod }}}{4 \pi \varepsilon_{0}}\left(\ln \left(\frac{1}{\left(S+d_{\text {LOM }}+x\right)^{2}}\right)+\ln \left(\frac{1}{\left(S+d_{\text {LOM }}-x\right)^{2}}\right)\right. \\
\left.-\ln \left(\frac{1}{\left(2 W+S-d_{L O M}+x\right)^{2}}\right)-\ln \left(\frac{1}{\left(2 W+S-d_{L O M}-x\right)^{2}}\right)\right) \\
=\frac{\lambda_{\text {rod }}}{4 \pi \varepsilon_{0}} \ln \left(\frac{\left(\left(2 W+S-d_{\text {LOM }}\right)^{2}-x^{2}\right)^{2}}{\left(\left(S+d_{\text {LOM }}\right)^{2}-x^{2}\right)^{2}}\right)
\end{gathered}
$$

where $x=0$ is defined as the centre of the cavity. Similarly, for the deflecting mode, the potential can be expressed as

$$
\begin{aligned}
V_{\text {dipole }} & =\frac{\lambda_{\text {rod }}}{4 \pi \varepsilon_{0}}\left(-\ln \left(\frac{1}{\left(S+d_{\text {dipole }}+x\right)^{2}}\right)+\ln \left(\frac{1}{\left(S+d_{\text {dipole }}-x\right)^{2}}\right)\right. \\
+ & \left.\ln \left(\frac{1}{\left(2 W+S-d_{\text {dipole }}+x\right)^{2}}\right)-\ln \left(\frac{1}{\left(2 W+S-d_{\text {dipole }}-x\right)^{2}}\right)\right) \\
= & \frac{\lambda_{\text {rod }}}{4 \pi \varepsilon_{0}} \ln \left(\frac{\left(2 W+S-d_{\text {dipole }}+x\right)^{2}\left(S+d_{\text {dipole }}-x\right)^{2}}{\left(2 W+S-d_{\text {dipole }}-x\right)^{2}\left(S+d_{\text {dipole }}+x\right)^{2}}\right) .
\end{aligned}
$$

The potentials for the LOM and deflecting mode are firstorder approximations because the exact potentials would require evaluating an infinite number of image charges, although the approximation is accurate to $\leq 5 \%$ if $W, S>2 R$. To determine the apparent displacement of the line charges for the LOM and deflecting modes, we require that the potential around the rod be uniform, thus the potential potential is equal at $x=S \pm R$. For the LOM and deflecting modes respectively, we obtain the line charge displacements as

$$
\begin{gathered}
d_{L O M}=W-\sqrt{\alpha+R^{2}-2 \sqrt{R^{2} \alpha+\beta^{2}}} \\
d_{\text {dipole }}=W-\sqrt{\alpha-3 R^{2}-2 \sqrt{2 R^{4}-\alpha R^{2}+\beta^{2}}}
\end{gathered}
$$

where $\alpha$ and $\beta$ are

$$
\begin{gathered}
\alpha=S^{2}+(S+W)^{2} \\
\beta=S(S+W)
\end{gathered}
$$

Although the potentials in Eqs. 6 and 7 have been derived by considering only the first two image charges. As there will also be image charges of the image charges themselves, we end up with an infinite sum of terms. However, as the higher order terms diminish with increasing order, we can truncate the series with little affect on accuracy. The solution to arbitrary order $N$ is

$$
V(x)=\frac{\lambda_{\text {rod }}}{4 \pi \varepsilon_{0}} \sum_{n=1}^{N} \ln \left(\varphi_{n}(x)\right)=\frac{\lambda_{\text {rod }}}{4 \pi \varepsilon_{0}} \ln \left(\prod_{n=1}^{N} \varphi_{n}(x)\right)
$$

where $\varphi_{n}$ for the LOM and deflecting potentials respectively can be expressed as

$$
\begin{gathered}
\varphi_{\text {LOM }, n}=\left(\frac{\left(\left(2 n W+(2 n-1) S-d_{L O M}\right)^{2}-x^{2}\right)^{2}}{\left(\left(2(n-1) W+(2 n-1) S+d_{L O M}\right)^{2}-x^{2}\right)^{2}}\right)^{(-1)^{n-1}} \\
\varphi_{\text {dipole }, n}=\frac{\left(2 n W+(2 n-1) S-d_{\text {dipole }}+x\right)^{2}\left(2(n-1) W+(2 n-1) S+d_{\text {dipole }}-x\right)^{2}}{\left(2 n W+(2 n-1) S-d_{\text {dipole }}-x\right)^{2}\left(2(n-1) W+(2 n-1) S+d_{\text {dipole }}+x\right)^{2}}
\end{gathered}
$$

The expressions for $d_{L O M}$ and $d_{\text {dipole }}$ given in Eqs. 8 and 9 are solutions to the $4^{\text {th }}$-order polynomials obtained by solving $\sqrt{\varphi_{1}(S+R)}+\sqrt{\varphi_{1}(S-R)}=0$; of which only one of the 


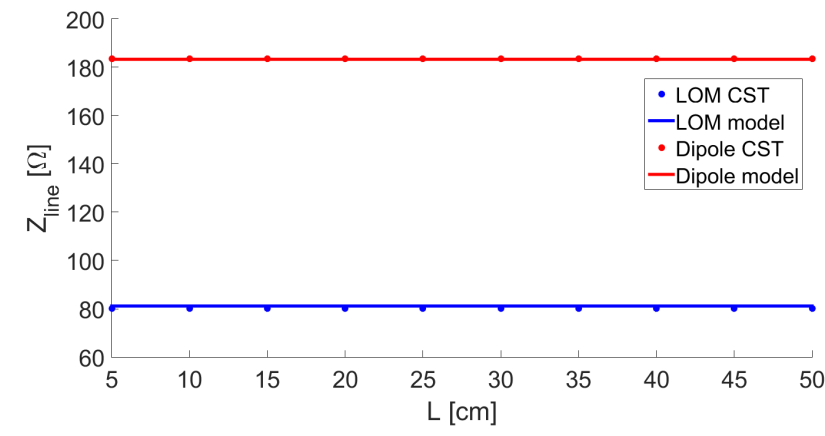

Figure 5: Scans of line impedance vs rod length, $L_{\text {rod }}$, comparing model (solid lines) to results from CST (points) for the LOM (blue) and deflecting (red) modes.

four possible solutions to each equation is physical. To evaluate $d_{L O M}$ and $d_{\text {dipole }}$ to second order or higher, the solution must be solved numerically as no analytical solution exists for higher order approximations. As only one of the $4 n$ solutions to the polynomial gives a physical solution, when evaluating $d_{L O M}$ and $d_{\text {dipole }}$ to $n^{\text {th }}$-order numerically, the first order approximations from Eqs. 8 and 9 should be used as the initial values.

Having determined the potential for the LOM and deflecting mode, we can determine the capacitance per unit length of the transmission line as

$$
C_{\text {line }}=\frac{\lambda_{\text {rod }}}{V} .
$$

Thus the characteristic impedance of the transmission line can be defined as

$$
Z_{0}=\frac{1}{c C_{\text {line }}}=\frac{V}{c \lambda_{\text {rod }}}
$$

where $c$ is the speed of light in a vacuum. Since the capacitances of the LOM combine in parallel and for the deflecting mode they combine in series, the characteristic line impedance for the LOM and deflecting mode respectively are

$$
Z_{0, x}^{L O M}=\frac{1}{8 \pi \varepsilon_{0} c} \ln \left(\prod_{n=1}^{N} \varphi_{L O M, n}(S+R)\right)
$$

and

$$
Z_{0, x}^{\text {dipole }}=\frac{1}{2 \pi \varepsilon_{0} c} \ln \left(\prod_{n=1}^{N} \varphi_{\text {dipole }, n}(S+R)\right) .
$$

Figures 5-8 show parameter scans of line impedance vs. deflecting cavity geometry parameters for both the LOM and deflecting mode for rod length, $L_{\text {rod }}$, transverse rod half separation, $S$, rod to wall separation, $W$, and rod radius, $R$, respectively, comparing results from CST [12] to the models given in Eqs. 15 and 16 evaluated using the first $10^{5}$ terms. As we have assumed that the rods are infinitely long, the line impedance is not dependent on $L_{\text {rod }}$, similarly, the longitudinal rod half separation, $g$, only affects the geometry near the end of the rod and will not affect the line impedance.

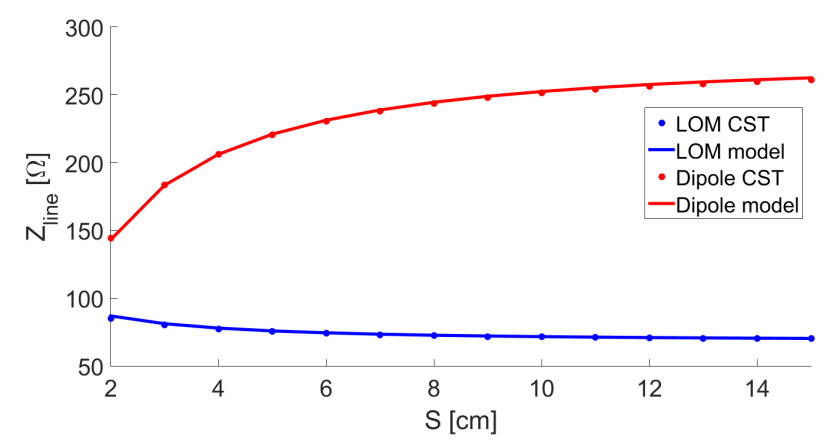

Figure 6: Scans of line impedance vs transverse rod half separation, $S$, comparing model (solid lines) to results from CST (points) for the LOM (blue) and deflecting (red) modes.

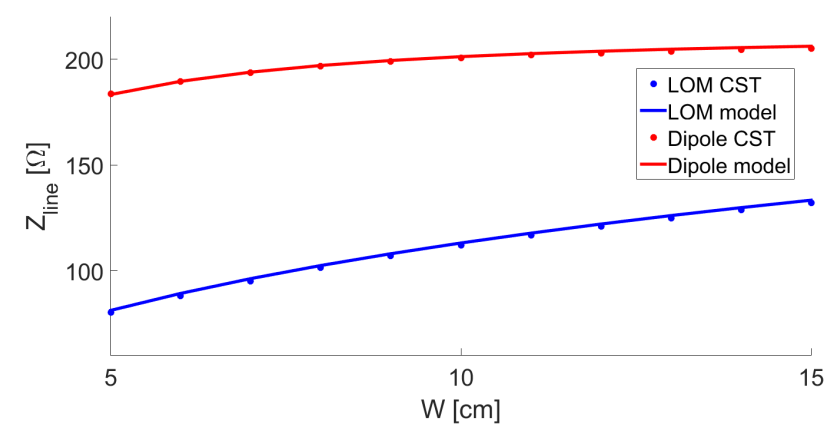

Figure 7: Scans of line impedance vs rod to wall separation, $W$, comparing model (solid lines) to results from CST (points) for the LOM (blue) and deflecting (red) modes.

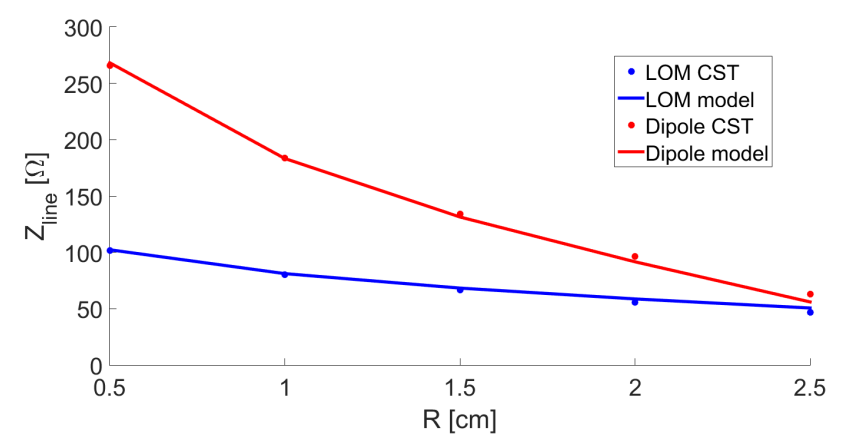

Figure 8: Scans of line impedance vs rod radius, $R$, comparing model (solid lines) to results from CST (points) for the LOM (blue) and deflecting (red) modes. 

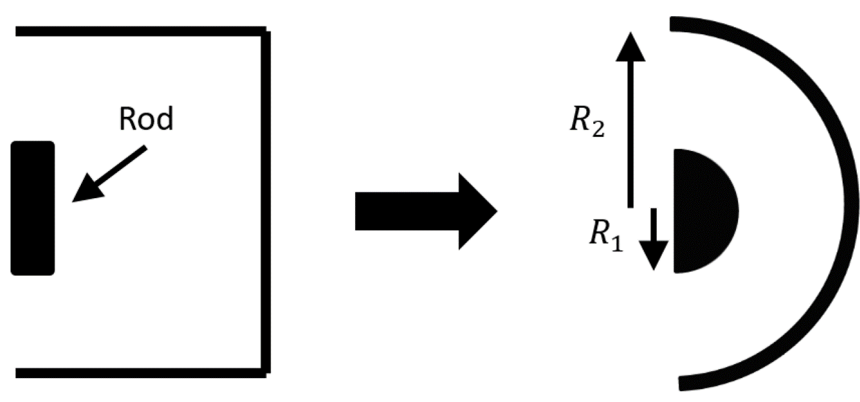

Figure 9: A diagram showing the geometric similarity between the end capacitance geometry and a hemispherical capacitor.

From Figure 8, as the rod radius increases, the accuracy of the model deteriorates; the same is also true in Figures 6 and 7 as $S$ and $W$ decrease. This is because our model neglects image charges in the rods as these will affect the displacements, $d_{L O M}$ and $d_{\text {dipole }}$, of the line charge in the rod. When $R \ll S, W$ these image charges have a negligible effect on $d_{L O M}$ and $d_{\text {dipole }}$, however, if $R$ becomes comparable to $S$ or $W$ then these image charges will significantly affect $d_{L O M}$ and $d_{\text {dipole }}$.

\subsection{End Capacitance}

The end capacitance is difficult to model because the electric field in the capacitive gap is strongly dependent on all the design parameters of the 4RDC. We shall model the end capacitance by starting with a simple physical model and then modify this by adding empirical correction terms to obtain a model in good agreement with CST [12]. By considering the symmetry planes in the cavity for the LOM and deflecting modes (Figure 3), we can simplify the geometry of the cavity to provide an electrically equivalent geometry to calculate the end capacitance. For the LOM, the two transversely opposing rods have the same potential and no capacitance between them; therefore we can combine these two rods into one. For the deflecting plane, there is a symmetry plane between the transverse rods, thus we need only consider one rod. The model of the end capacitance can be considered as a hemispherical capacitor (Figure 9).

The capacitance of a hemispherical capacitor can be expressed as

$$
C=\frac{2 \pi \varepsilon_{0}}{\frac{1}{R_{1}}-\frac{1}{R_{2}}}
$$

where $R_{1}$ is the radius of the inner hemisphere and $R_{2}$ the radius of the outer hemisphere. For the end capacitance, we shall assume that $R_{1}=R$ and $R_{2}=R+g$, thus the capacitance of the hemispherical capacitor can be expressed as

$$
C=\frac{2 \pi \varepsilon_{0}}{\frac{1}{R}-\frac{1}{R+g}}=\frac{2 \pi \varepsilon_{0} R^{2}}{g}+2 \pi \varepsilon_{0} R .
$$

Eq. 18 does not describe the end capacitance, but it is the first step towards our model. The first term in Eq 18 is very similar to the equation for a parallel plate capacitor and depends on the geometry of the capacitor, whereas the second term is the capacitance of the inner hemisphere, or in our case rod, in the absence of the outer sphere, this is often referred to as the selfcapacitance. The end capacitance is strongly dependent on the fringe fields in the capacitive gap, therefore we shall replace the geometric capacitance term from Eq. 18 with a capacitance model for a parallel plate capacitor which includes fringe fields [15]. The capacitance for the parallel plate capacitor is

$$
C_{P P}=\frac{\pi \varepsilon_{0} R^{2}}{g}\left[1+\frac{g}{\pi R}\left(1+\ln \left(\frac{2 \pi R}{g}\right)\right)\right] .
$$

For our model of the end capacitance, $C_{\text {end }}$, we shall consider a parallel plate capacitor with some correction terms; these terms are empirically derived as the capacitance for such a complex system cannot be easily derived. We shall consider the end capacitance to have the following form

$$
C_{\text {end }}=C_{P P} f_{L}\left(f_{S}+f_{W}\right)+f_{R}+f_{g} .
$$

In Eq. 20, $C_{P P}$ is the capacitance of a parallel plate capacitor and is dependent on the geometry of the system; this term will also depend on $L_{\text {rod }}, S$ and $W$ and hence correction terms, $f_{L}$, $f_{S}$ and $f_{W}$ respectively are included. $f_{R}$ and $f_{g}$ are additional correction terms depending on $R$ and $g$ respectively which can be considered the self-capacitance of the rod. We have chosen $f_{L}$ to have the form

$$
f_{L}=\frac{a_{L 1}}{\sqrt{1+a_{L 2}\left(\frac{L}{L_{0}}\right)^{a_{L 3}}}}+1-\frac{a_{L 1}}{\sqrt{1+a_{L 2}}}
$$

where $a_{L i}$ are fitting parameters, $L_{0}$ is a reference value for $L_{r o d}$ and $1-\frac{a_{L 1}}{\sqrt{1+a_{L 2}}}$ is used to ensure that $f_{L}=1$ when $L_{\text {rod }}=L_{0}$. Similarly for $f_{S}$ and $f_{W}$ we have chosen the form

$$
\begin{aligned}
f_{S}+f_{W} & =\frac{a_{S 1}}{\sqrt{1+a_{S 2}\left(\frac{S}{S_{0}}\right)^{a_{S 3}}}}+\frac{a_{W 1}}{\sqrt{1+a_{W 2}\left(\frac{W}{W_{0}}\right)^{a_{W 3}}}} \\
& +1-\frac{a_{S 1}}{\sqrt{1+a_{S 2}}}-\frac{a_{W 1}}{\sqrt{1+a_{W 2}}}
\end{aligned}
$$

This functional form has been chosen for $f_{L}, f_{S}$ and $f_{W}$ because it can be fit to a wide range of functions depending on the values of the fit parameters. For the self-capacitance terms, we have assumed a function of the form

$$
f_{R}+f_{g}=a_{R} \pi \varepsilon_{0} R+a_{g 1}\left(\frac{g}{g_{0}}\right)^{a_{g 2}}
$$

By fitting Eq. 20 to the results of CST simulations, using the functional forms for the correction terms given in Eqs. 2123 and combining the reference parameters with the associated fit coefficients, we obtain end capacitances for the LOM and deflecting mode respectively as 


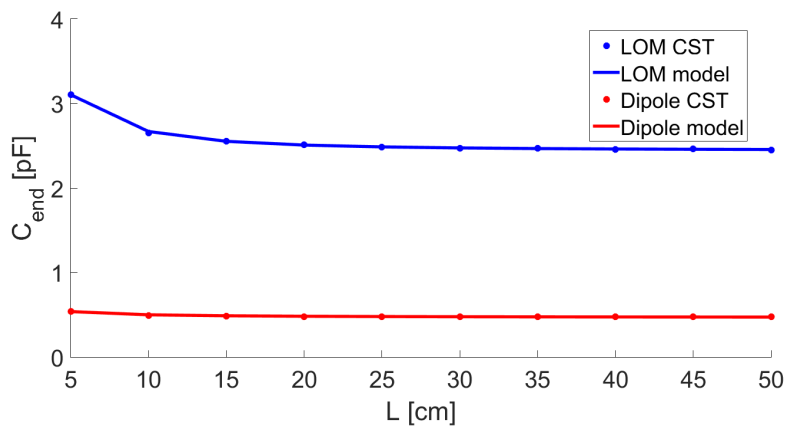

Figure 10: Scans of end capacitance vs rod length, $L_{\text {rod }}$, comparing model (solid lines) to results from CST (points) for the LOM (blue) and deflecting (red) modes.

$$
\begin{gathered}
C_{\text {end }}^{L O M}=\pi \varepsilon_{0}\left[\frac{2 R^{2}}{g}\left[1+\frac{g}{\pi R}\left(1+\ln \left(\frac{2 \pi R}{g}\right)\right)\right] \times\right. \\
\left(\frac{1.05}{\sqrt{1+1.1 \times 10^{5} L_{\text {rod }}^{3.68}}}+0.90\right)\left(12.14-\frac{5.56}{\sqrt{1+4.02 S^{0.73}}}-\frac{7.14}{\sqrt{1+8.79 W^{1.14}}}\right)+ \\
\left.\left(1.97 R+2.93 \times 10^{-3} g^{-0.53}\right)\right]
\end{gathered}
$$

and

$$
\begin{gathered}
C_{\text {end }}^{\text {dipole }}=\pi \varepsilon_{0}\left[\frac{R^{2}}{2 g}\left[1+\frac{g}{\pi R}\left(1+\ln \left(\frac{2 \pi R}{g}\right)\right)\right] \times\right. \\
\left(\frac{3.63}{\sqrt{1+1.6 \times 10^{5} L_{\text {rod }}^{2.26}}}+0.92\right)\left(8.44-\frac{6.00}{\sqrt{1+5.19 S^{0.52}}}-\frac{6.49}{\sqrt{1+8.68 W^{0.29}}}\right)+ \\
\left.\left(0.21 R+1.56 \times 10^{-3} g^{-0.29}\right)\right]
\end{gathered}
$$

It should be noted that the end capacitances of the longitudinally opposed rods are combined in parallel for the LOM and in series for the deflecting term; thus the first term varies as $\frac{2 R^{2}}{g}$ for the LOM and $\frac{R^{2}}{2 g}$ for the deflecting mode. Although these equations were empirically derived, the form from Eq. 20 is physically justified. The mutual capacitance term, described as a parallel plate capacitor with fringe fields, will be dependent on the geometry of the system and will therefore depend on all the design parameters. The self-capacitance term will depend on the shape of the conductor and not on the local geometry. The functional forms of the correction terms were chosen to allow a good fit to a complicated function.

Figures 10-14 show parameter scans of end capacitance vs. deflecting cavity geometry parameters for both the LOM and deflecting mode for $L_{\text {rod }}, S, W, R$ and $g$, respectively, comparing results from CST [12] to the models given in Eqs. 24 and 25.

\subsection{Resonant Frequency}

Having determined models for the line impedance and end capacitance, Eq. 2 can be solved numerically to determine the

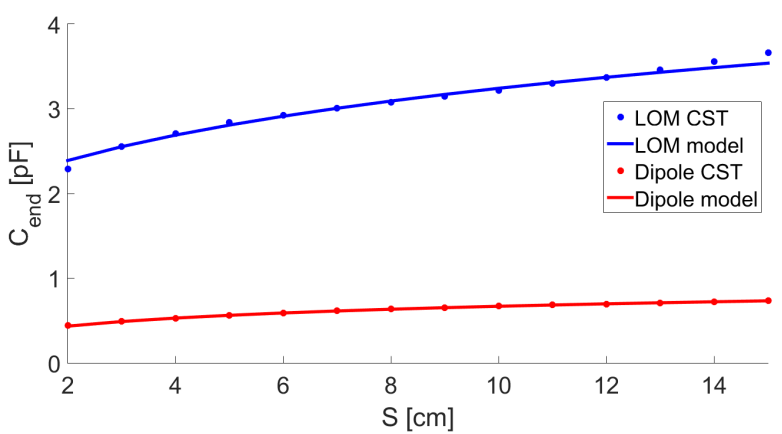

Figure 11: Scans of end capacitance vs transverse rod half separation, $S$, comparing model (solid lines) to results from CST (points) for the LOM (blue) and deflecting (red) modes.

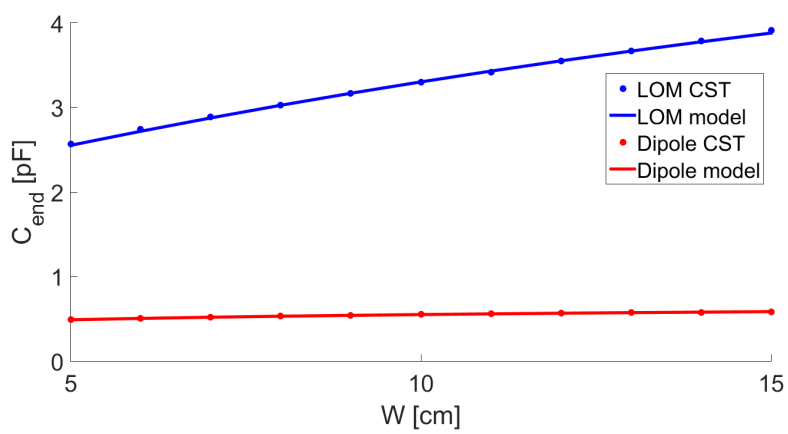

Figure 12: Scans of end capacitance vs rod to wall separation, $W$, comparing model (solid lines) to results from CST (points) for the LOM (blue) and deflecting (red) modes.

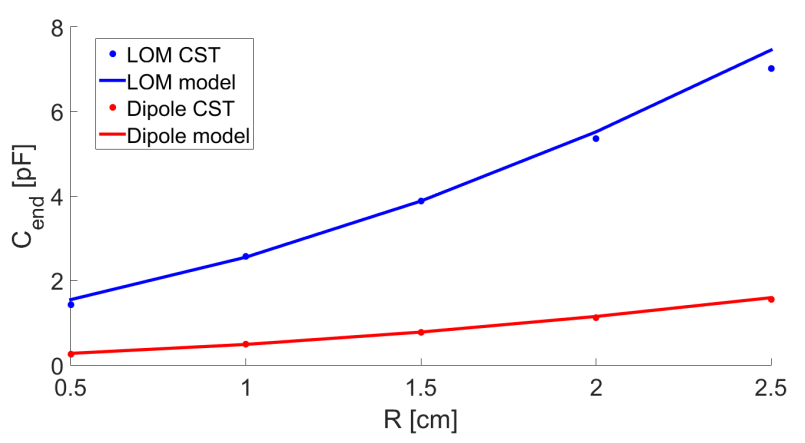

Figure 13: Scans of end capacitance vs rod radius, $R$, comparing model (solid lines) to results from CST (points) for the LOM (blue) and deflecting (red) modes. 


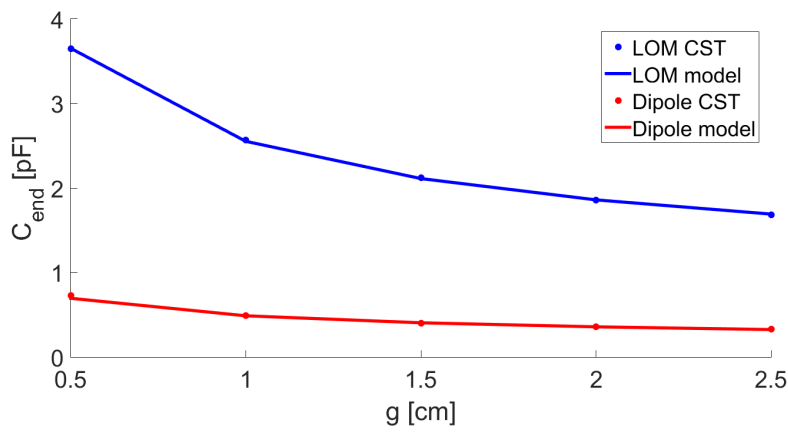

Figure 14: Scans of end capacitance vs longitudinal rod half separation, $g$, comparing model (solid lines) to results from CST (points) for the LOM (blue) and deflecting (red) modes.

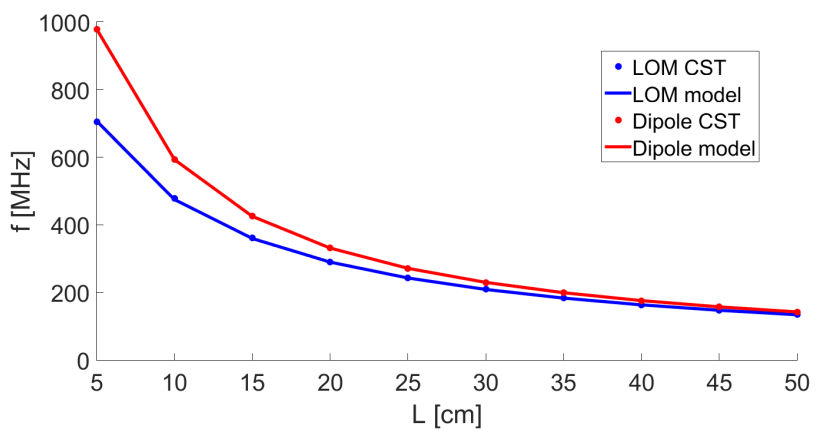

Figure 15: Scans of resonant frequency vs rod length, $L_{\text {rod }}$, comparing model (solid lines) to results from CST (points) for the LOM (blue) and deflecting (red) modes.

resonant frequency of the LOM and deflecting mode. Figures 15-19 shows parameter scans of the resonant frequency vs. deflecting cavity design parameters for the LOM and deflecting mode, comparing results from CST [12] to the model described within this report. Due to limitations in the models of line impedance and end capacitance, the modeled frequency vs $R$ deviates from the CST results for large values of $R$, however the errors are $>10 \%$ and are still capable of providing a good estimate for the resonant frequencies of each mode.

\subsection{Modeling the transverse geometric shunt impedance, $\frac{R_{T}}{Q}$}

$\frac{R_{T}}{Q}$ is an essential figure of merit for an cavity as this quantifies how strongly the fields in the cavity couple to the beam. Using an approach similar to that outlined in [11], we shall derive an expression for the $\frac{R_{T}}{Q}$ for the deflecting mode of a four-rod deflecting cavity and compare it to CST simulations. The model derived in this section is an improvement to that described in [11] as the authors neglect the end capacitance between longitudinally opposing rods whereas we take this into account.

We shall assume that the potential along the rod is distributed as

$$
V=V_{0} \sin \left(\frac{2 \pi z}{\lambda}\right)
$$

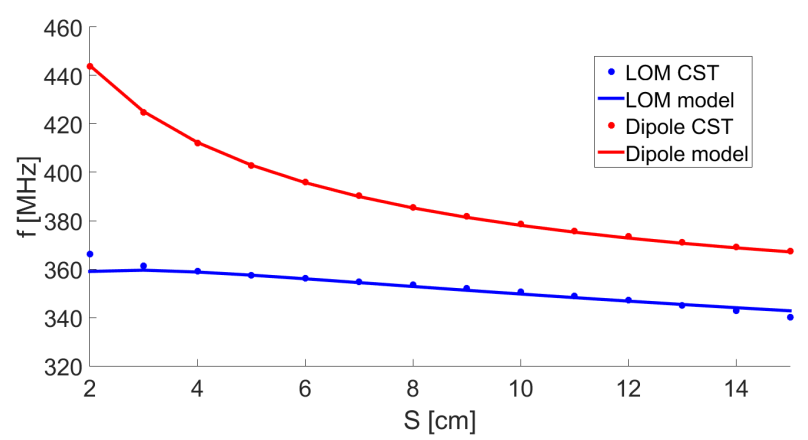

Figure 16: Scans of resonant frequency vs transverse rod half separation, $S$, comparing model (solid lines) to results from CST (points) for the LOM (blue) and deflecting (red) modes.

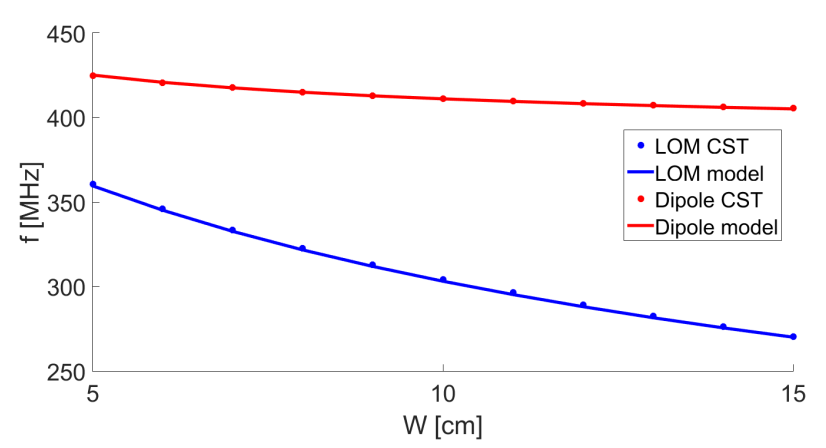

Figure 17: Scans of resonant frequency vs rod to wall separation, $W$, comparing model (solid lines) to results from CST (points) for the LOM (blue) and deflecting (red) modes.

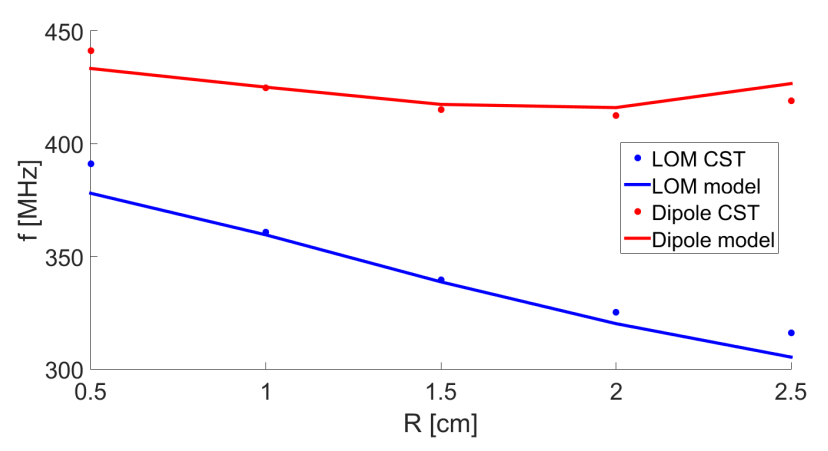

Figure 18: Scans of resonant frequency vs rod radius, $R$, comparing model (solid lines) to results from CST (points) for the LOM (blue) and deflecting (red) modes. 


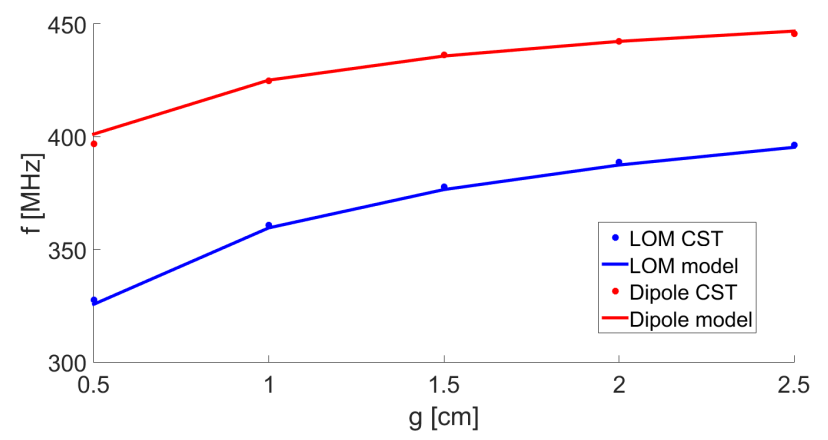

Figure 19: Scans of resonant frequency vs longitudinal rod half separation, $g$, comparing model (solid lines) to results from CST (points) for the LOM (blue) and deflecting (red) modes.

where we assume that the wavelength, $\lambda=4\left(L_{r o d}+g\right)$. This is an approximation because some of the field penetrates into the beam pipe, so the actual wavelength is slightly larger than $4\left(L_{\text {rod }}+g\right)$, but this is a good approximation if $L_{\text {rod }} \gg 0$. The the region between the end of the rod and the midplane of the cavity, we assume that the potential drops off linearly to zero, therefore the potential along the axis of one of the rods in the region $0 \leq z \leq L_{\text {rod }}+g$ is given as

$V= \begin{cases}V_{0} \sin \left(\frac{\pi z}{2\left(L_{\text {rod }}+g\right)}\right) & \text { if } z \leq L_{\text {rod }} \\ \frac{V_{0}}{g} \sin \left(\frac{\pi L_{\text {rod }}}{2\left(L_{\text {rod }}+g\right)}\right)\left(\left(L_{\text {rod }}+g\right)-z\right) & \text { if } L_{\text {rod }}<z \leq\left(L_{\text {rod }}+g\right)\end{cases}$

The energy stored in one rod in the cavity is a quarter of the energy stored in the cavity, thus the total energy stored is

$$
U_{\text {stored }}=4\left(\int_{0}^{L_{\text {rod }}} C_{\text {line }} V^{2} d z+\int_{L_{\text {rod }}}^{L_{\text {rod }}+g} \frac{C_{\text {end }}}{g} V^{2} d z\right) .
$$

Using Eqs. 14 and 27 and solving the integrals, the stored energy is given by

$U_{\text {stored }}=2 V_{0}^{2}\left[\left(\frac{L_{\text {rod }}}{Z_{0} c}+\frac{C_{\text {end }}}{3}\right)-\left(\frac{L_{\text {rod }}+g}{\pi Z_{0} c}+\frac{C_{\text {end }}}{3}\right) \sin \left(\frac{\pi L_{\text {rod }}}{L_{\text {rod }}+g}\right)\right]$.

The $\frac{R_{T}}{Q}$ is given by

$$
\frac{R_{T}}{Q}=\frac{1}{\omega U_{\text {stored }}}\left(\frac{\nabla_{\perp} V}{k}\right)^{2}
$$

where $k=\frac{2 \pi}{\lambda}=\frac{\omega}{c}$. Therefore the $\frac{R_{T}}{Q}$ can be expressed as

$$
\frac{R_{T}}{Q}=\frac{c^{2}}{2 \omega^{3}\left[\left(\frac{L_{\text {rod }}}{Z_{0} c}+\frac{C_{\text {end }}}{3}\right)-\left(\frac{L_{\text {rod }}+g}{\pi Z_{0} c}+\frac{C_{\text {end }}}{3}\right) \sin \left(\frac{\pi L_{\text {rod }}}{L_{\text {rod }}+g}\right)\right]}\left(\frac{\nabla_{\perp} V}{V_{0}}\right)^{2} .
$$

If we use Eqs. 11 and 12 to evaluate the potential at a small transverse offset, $x$, and by symmetry, the potential along the longitudinal axis of the cavity is zero, then

$$
\frac{\nabla_{\perp} V}{V_{0}}=\frac{V(x)}{x V_{0}}
$$

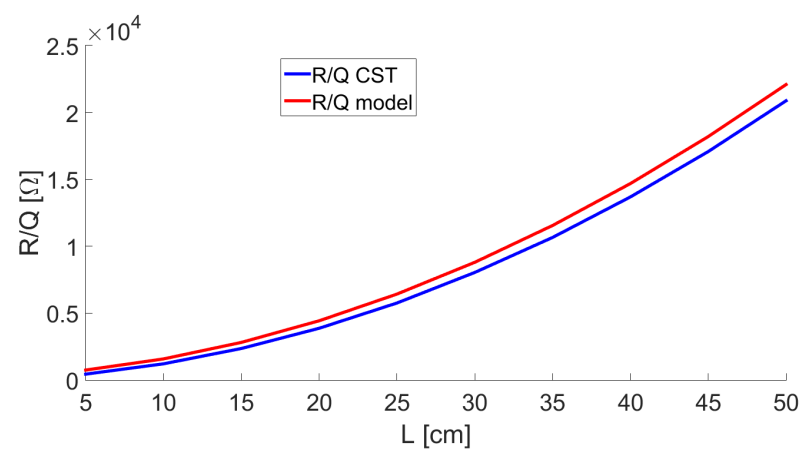

Figure 20: Scans of $\frac{R_{T}}{O}$ vs rod length, $L_{\text {rod }}$, comparing the equivalent circuit model (red) to results from CST (blue) for the deflecting mode.

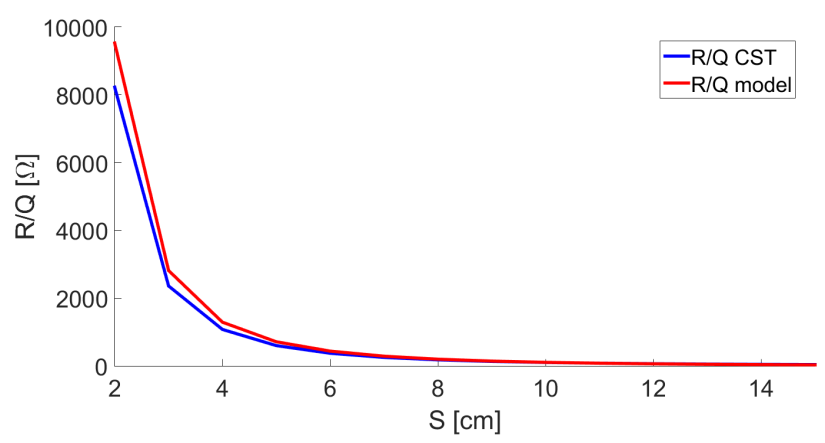

Figure 21: Scans of $\frac{R_{T}}{Q}$ vs transverse rod half separation, $S$, comparing the equivalent circuit model (red) to results from CST (blue) for the deflecting mode.

where $V(x)$ is the potential evaluated at a small transverse offset $x$ and $V_{0}$ is the potential evaluated at the surface of the rod. Note that in Eq. 32, we assume that the potential varies linearly with $x$, although this is only valid for small values of $x$. By taking this approximation, $\frac{R_{T}}{Q}$ becomes

$$
\frac{R_{T}}{Q}=\frac{c^{2}}{2 \omega^{3}\left[\left(\frac{L_{\text {rod }}}{Z_{0} c}+\frac{C_{\text {end }}}{3}\right)-\left(\frac{L_{\text {rod }}+g}{\pi Z_{0} c}+\frac{C_{\text {end }}}{3}\right) \sin \left(\frac{\pi L_{\text {rod }}}{L_{\text {rod }}+g}\right)\right]}\left(\frac{V(x)}{x V_{0}}\right)^{2}
$$

which is an equation which only depends on the cavity design parameters $\left(L_{\text {rod }}\right.$ and $g$ ) and on parameters which have been derived in previous sections $\left(C_{\text {end }}, Z_{0}, \omega\right.$ and $\left.V(x)\right)$.

Figures 20-24 show scans of $\frac{R_{T}}{Q}$ vs different design parameters, comparing the model and CST results. With all of these figures, it is clear that the model describes the trend of $\frac{R_{T}}{Q}$ vs the different design parameters very well, however there is a systematic error, which is most clearly seen on Figures 22 and 24. This systematic error is due to the fact that we assume a simplistic model for the potential along the rod and between the rod and the grounding plane; this affects the stored energy of the cavity, thus $\frac{R_{T}}{Q}$. However, in order to optimise a cavity design, maximising $\frac{R_{T}}{Q}$ is generally more important than the exact value of $\frac{R_{T}}{Q}$. 


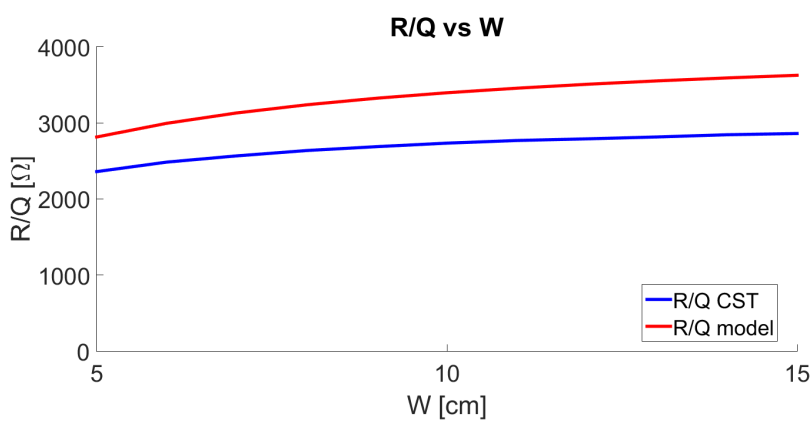

Figure 22: Scans of $\frac{R_{T}}{Q}$ vs rod to wall separation, $W$, comparing the equivalent circuit model (red) to results from CST (blue) for the deflecting mode.

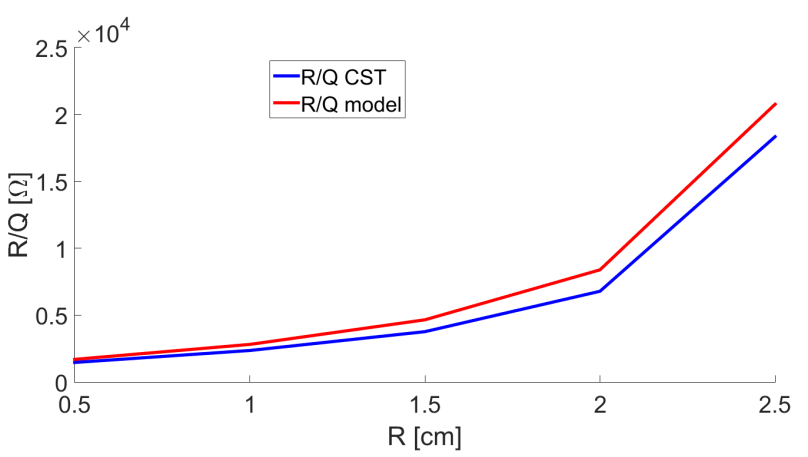

Figure 23: Scans of $\frac{R_{T}}{O}$ vs rod radius, $R$, comparing the equivalent circuit model (red) to results from CST (blue) for the deflecting mode.

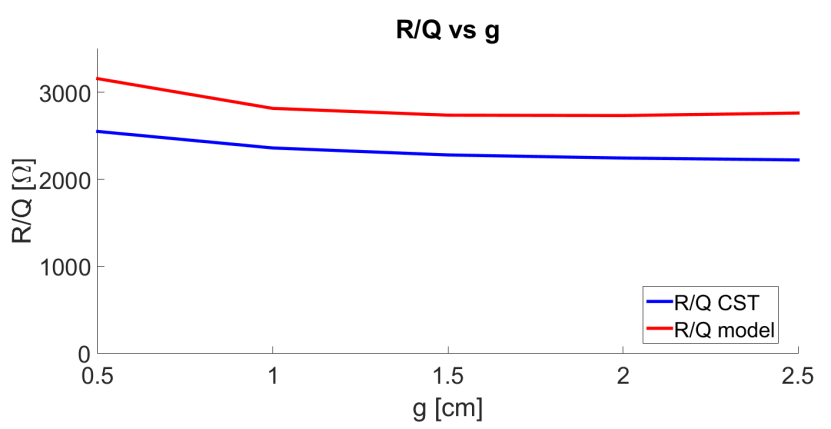

Figure 24: Scans of $\frac{R_{T}}{Q}$ vs longitudinal rod half separation, $g$, comparing the equivalent circuit model (red) to results from CST (blue) for the deflecting mode.

\subsection{Comparison Between the Improved Equivalent Circuit Model and the CEBAF and HL-LHC deflecting cavities}

As a benchmark we compare the simulations and calculations for the two existing 4 rod deflecting cavities. These two cavities make an effective benchmark as the rod radius and separation compared to the wall radius is very different and hence verifies our model in the two extremes. Table 1 shows the design parameters for the CEBAF separator [6] and the HL-LHC four-rod crab cavity [8] and shows a good agreement between the simulations in CST and the prediction from the equivalent circuit model. The CST models of the CEBAF and HL-LHC cavities are simplified versions of the actual designs described in $[6,8]$ in order to compare to the equivalent circuit model.

Table 1: A table of parameters for the CEBAF separator [6]

\begin{tabular}{|c|c|c|c|c|}
\hline Parameter & \multicolumn{2}{|c|}{ CEBAF } & \multicolumn{2}{c|}{ HL-LHC } \\
\hline$L_{\text {rod }}[\mathrm{cm}]$ & \multicolumn{2}{|c|}{12.8} & \multicolumn{2}{c|}{16.3} \\
\hline$R[\mathrm{~cm}]$ & \multicolumn{2}{|c|}{1} & \multicolumn{2}{c|}{2} \\
\hline$S[\mathrm{~cm}]$ & \multicolumn{2}{|c|}{1.75} & \multicolumn{2}{c|}{4} \\
\hline$W[\mathrm{~cm}]$ & \multicolumn{2}{|c|}{12.85} & \multicolumn{2}{c|}{3} \\
\hline$g[\mathrm{~cm}]$ & \multicolumn{2}{|c|}{15} & CST & Model \\
\hline Frequencies $[\mathrm{MHz}]$ & CST & Model & 366.4 & 362.8 \\
\hline LOM & 320.9 & 307.1 & 394.1 & 390.6 \\
\hline Deflecting & 507.7 & 505.3 & 411.1 & 459.8 \\
\hline $2^{\text {nd }}$ monopole & 546.3 & 585.5 & 408.1 & 459.8 \\
\hline $2^{\text {nd }}$ dipole & 552.9 & 585.5 & 40.2 & 580.0 \\
\hline$\frac{R_{T}}{Q}$ & 10941 & 15017 & 464.2 & \\
\hline
\end{tabular}

For the deflecting modes in Table 1, CST and the equivalent circuit model agree within $0.47 \%$ for the CEBAF separator and within $0.89 \%$ for the HL-LHC four-rod crab cavity. For the LOMs, CST and the equivalent circuit model agree within $4.3 \%$ for the CEBAF separator and within $0.93 \%$ for the HLLHC four-rod crab cavity. Since we neglect end capacitance for the $2^{\text {nd }}$ monopole and dipole modes, we assume that both frequencies are that for a quarter-wave resonator and the model and CST agree for these modes within 7\% for the CEBAF separator and within $12 \%$ for the HL-LHC crab cavity. Due to the simplicity of the model of the $2^{\text {nd }}$ monopole and dipole modes, we do not expect a good agreement between model and simulation.

Design specifications stated in the HiLumi LHC Final Project Report [16] places constraints on the cavity dimensions and beam impedance; which in turn defines the values for $S$ and $W$. In order to separate the LOM and deflecting frequencies, we require a small value of $S$ and a large value of $W$, however for the HL-LHC crab cavity we have a large value of $S$ and a small value of $W$; therefore an alternative method of separating frequencies is needed. The HL-LHC crab cavity has been designed with complex rod shapes in order to reduce the capacitance to the outer wall and increase the capacitance to the transversely opposing rods in order to mimic the effect of reducing $S$ and increasing $W$. If the LOM and deflecting mode cannot be separated sufficiently, LOM and HOM (higher order mode) couplers can be used to damp unwanted modes [17]. 


\subsection{Design optimisation with the equivalent circuit model}

Design specifications constrain the design parameters for the cavity. In the case of the HL-LHC crab cavity the stringent design specifications limit the possible range of the design parameters. For the HL-LHC the specifications are

1. cavity aperture: $S-R=42 \mathrm{~mm}$

2. transverse cavity size: $S+W=102 \mathrm{~mm}$

3. deflecting mode frequency: $f=400 \mathrm{MHz} \propto \frac{1}{L_{\text {rod }}}$

4. maximise mode separation: maximise $R, W$, minimise $S$

5. maximise $\frac{R_{T}}{Q}$ : maximise $R, W$, minimise $S, g$

Since the deflecting mode frequency is most strongly dependent on $L_{\text {rod }}$, we shall assume that this specification constrains $L_{\text {rod }}$. In addition to the above constraints, the peak surface fields need to be considered. An equivalent circuit model cannot estimate peak surface fields, but a study in CST for the HL-LHC crab cavity determined that we require $W-R \leq 20 \mathrm{~mm}$ and $g \geq 30 \mathrm{~mm}$ to keep peak fields within acceptable limits. From the fifth specification above, we need to minimise $g$, therefore $g=30 \mathrm{~mm}$ is optimal. Since we need to maximise $R$, we can state that $W-R=20 \mathrm{~mm}$, which combined with the first 2 specifications above gives $S=62 \mathrm{~mm}, W=40 \mathrm{~mm}$ and $R=20 \mathrm{~mm}$. Then $L_{\text {rod }}$ is determined to provide the correct resonant frequency.

In the case of the CEBAF deflector, there was no limit on the transverse cavity size, hence in the CEBAF design, this was made significantly larger to increase $\frac{R_{T}}{Q}$ and mode separation. As the CEBAF separator is a CW normal conducting cavity, it is power limited and operates at a lower transverse voltage, thus peak surface electric field is less critical and $g=10 \mathrm{~mm}$.

In general, we can assume that $L_{\text {rod }}$ is used to obtain the correct resonant frequency of the deflecting mode and as we aim to maximise mode separation and $\frac{R_{T}}{Q}$, we need to maximise $R$ and $W$, while minimising $S$ and $g$. Other design specifications, such as cavity aperture and limits on peak surface fields apply further limits to constrain the design parameters and allow an optimum or a compromise to be obtained.

\section{Summary}

An improved equivalent circuit model for a four rod deflecting cavity is presented in this paper. We present a physical model for the line impedance of the rods and a semi-empirical model for the end capacitance. From the line impedance and end capacitance we are able to calculate the resonant frequency of the deflecting cavity for the LOM and deflecting mode. Models of the line impedance, end capacitance and resonant frequency are compared to CST simulations and show a good agreement. We then apply this model to develop a model for the $\frac{R_{T}}{Q}$ for the deflecting mode. The model provides $\frac{R_{T}}{Q}$ to an accuracy of $\leq 37 \%$, which is sufficient for understanding how to optimise the cavity design.

The equivalent circuit model was used to calculate resonant frequencies for the LOM and deflecting modes for the CEBAF deflector and HL-LHC four-rod crab cavity and the results compared to CST simulations. For the deflecting mode, CST and the equivalent circuit model agree within $0.47 \%$ for the CEBAF separator and within $0.89 \%$ for the HL-LHC four-rod crab cavity. For the LOM, CST and the equivalent circuit model agree within $4.3 \%$ for the CEBAF separator and within $0.93 \%$ for the HL-LHC four-rod crab cavity.

The equivalent circuit model described in this paper assumes a simple geometry. It assumes cylindrical rods within a cuboid cavity. The cavity dimension is large out of the deflecting plane in order to neglect image charges out of the deflecting plane, we also neglect roundings on corners and edges as well as the effect of a beam pipe connected to the cavity.

Further studies are intended to obtain an improved model of the end capacitance. This model would be based on a more physically realistic model rather than an empirical fit to results from CST. This would allow for a model which is valid over a wider range of parameters.

\section{Acknowledgments}

The work undertaken in this paper was funded by the Cockcroft Institute Core Grant, HL-LHC-UK and HiLumi FP7. In addition, we would like to thank Dr Ben Hall and Dr Haipeng Wang for useful discussions related to this paper.

\section{References}

[1] S. Belomestnykh et al., (2010) "Deflecting cavity for beam diagnostics at Cornell ERL injector," Nucl. Instr. and Meth. A, Volume 614, Issue 2 (2010), pp. 179-183.

[2] M. Cornecchia and P. Emma, (2002) "Transverse to Longitudinal Emittance Exchange," SLAC-PUB-9225.

[3] M. Boorland, (2005) "Simulation and analysis of using deflecting cavities to produce short X-ray pulses with the Advanced Photon Source," Phys. Rev. ST Accel. Beams 8, 074001.

[4] K. Hosoyama et al., (2008) "Development of the KEK-B superconducting crab cavity," EPAC conference, pp. 2927-2931.

[5] G. Burt, (2010) "Transverse deflecting cavity lectures," CERN Accelerator School, 2010

[6] C. Hovater et al., (1996) "The CEBAF Separator System," JLAB-ACC-9618.

[7] O. Bruning and L. Rossi, (2015) "The High Luminosity Large Hadron Collider: The New Machine for Illuminating the Mysteries of Universe," World Scientific Publishing Co. Pte. Ltd., ISBN-13: 9789814675468.

[8] B. Hall, (2014) "Designing the four rod crab cavity for the high-luminosity LHC upgrade," EuCARD-BOO-2014-007, PhD Thesis.

[9] B. Xiao et al., (2015) "Design, prototyping, and testing of a compact superconducting double quarter wave crab cavity," Phys. Rev. ST Accel. Beams $18,041004$.

[10] S. U. De Silva and J. R. Delayen, (2013) "Design evolution and properties of superconducting parallel-bar rf-dipole deflecting and crabbing cavities," Phys. Rev. ST Accel. Beams 16, 012004.

[11] C. Leemann and C. G. Yao, (1990) "A Highly Effective Deflecting Structure".

[12] CST Microwave Studio.

[13] D. M. Pozar, (2005) "Microwave Engineering, 3 rd edition," John Wiley \& sons, Inc.

[14] I. Ben-Zvi and J. M. Brennen, (1983) "The quarter wave resonator as a superconducting linac element," Nucl. Instr. and Meth., Volume 212, Issue 1 (1983), pp. 73-79.

[15] H. B. Palmer, (1937) "The Capacitance of a Parallel-Plate Capacitor by the Schwartz-Christoffel Transformation".

[16] A. Barachetti et al. (HiLumi LHC collaboration), (2016) "HiLumi LHC Final Design Report," CERN-ACC-2016-0007. 
[17] G. Burt and H. Wang, (2014) "Damping of unwanted modes in SRF deflecting/crabbing cavities," Nucl. Instr. and Meth. A, Volume 734, Part A (2014), pp. 65-71. 